\title{
Positional relationships of abdominal aortic branches for contrast radiography of the inferior mesenteric artery using the coeliac trunk and superior mesenteric artery as landmarks
}

\author{
By \\ Yusuke NAKAYAMA ${ }^{1,2}$, Shogo HAYASHI ${ }^{2}$, Kyoko TAKEUCHI ${ }^{2,3}$, \\ Shinichi KAWATA ${ }^{2}$, Ning $\mathbf{Q U}^{2}$, and Masahiro ITOH $^{2}$ \\ ${ }^{1}$ Department of Emergency and Critical Care Medicine, Yokohama City Minato Red Cross Hospital, \\ 3-12-1 Shinyamashita, Naka-ku, Yokohama, Kanagawa 231-8682, Japan \\ ${ }^{2}$ Department of Anatomy, Tokyo Medical University, 6-1-1 Shinjuku, Shinjuku-ku, Tokyo 160-8402, Japan \\ ${ }^{3}$ Institute of Health Pedagogy Foundation, 203 Toshima-mansion, 3-2-7 Kanamecho, Toshima-ku, Tokyo 171-0043, Japan \\ -Received for Publication, December 13, 2016- \\ Key Words: thyroid gland follicles, macrophages, lymphocyte infiltration, immunohistochemistry, human elderly cadavers

\begin{abstract}
Summary: Purpose: To establish a method by which angiography of the inferior mesenteric artery (IMA) can be performed smoothly, we investigated the relative locations of the coeliac trunk (CT), superior mesenteric artery (SMA), IMA, and left renal artery (LtRA). Methods: From a total of 60 cadavers, 32 cadavers with few arteriosclerotic lesions and little vascular tortuosity were selected for the study. The abdominal aorta (Ao) were removed and incised on both lateral side, along the vertical axis and transected into the ventral and dorsal sides. The intravascular lumen on the ventral side of the Ao was photographed using a digital camera, and the horizontal and vertical diameters of the sites of confluence of the CT, SMA, and IMA, were measured on the computer screen. We also calculated the distances between the branches, including the CT, SMA, IMA, LtRA, and the common iliac artery (CoI). Results: Although the SMA-IMA distance did not correlate with the CT-SMA distance, the ratio of the SMA-IMA to CT-CoI distance was four times greater than the ratio of the CT-SMA to CT-CoI distance. Conclusions: The site of branching of the IMA can be inferred to some extent from the CT and SMA distance.
\end{abstract}

\section{Introduction}

Patients in the emergency room with gastrointestinal bleeding sometimes face the risk of death. In such cases, many physicians attempt treatment with colonoscopy. However, compared with upper gastrointestinal bleeding, colonoscopic treatment of lower gastrointestinal bleeding may sometimes be more difficult owing to residual material, such as feces. Such cases of lower gastrointestinal bleeding require rapid analysis by abdominal angiography, especially of the inferior mesenteric artery (IMA). When performing abdominal angiography, the coeliac trunk (CT) and superior mesenteric artery (SMA) tend to be easy to detect relative to the position of the diaphragm, spine, and liver parenchyma. In many cases, the renal artery is imaged based on its position relative to the renal parenchyma. As there are few organ landmarks in the vicinity of the IMA, the lumbar vertebrae are often used ${ }^{1}$. However, lumbar compression fractures and age-related changes in elderly patients cause vertebral deformities and spinal curvature, which often prevents the use of the spine as a landmark. Although it is possible to identify the location of blood vessels prior to angiography using 3D-CTA (three-dimensional computed tomography angiography) and other methods, reconstruction typically takes between 30 minutes to 1 hour, and hence these methods are not practical in emergency situations. Therefore, in this study, to reduce the time required for angiography of the IMA as well as to reduce patient radiation exposure, we investigated the relative positions of the branches of the IMA from the interior of the blood vessels, which are less likely to be affected by the thickness of the arterial walls and surrounding tissue. 


\section{Materials and Methods}

Sixty cadavers were investigated after the gross anatomy course at Tokyo Medical University in 2014 and 2015. Before their death, all donors signed a document agreeing to the donation of their bodies and their use for clinical stuidies. 28 were excluded owing to the presence of severe arteriosclerotic lesions and vascular tortuosity, and the remaining 32 cadavers (16 males and 16 females, aged 82.3 years) were analyzed. We first analyzed the association between the branches of the IMA and lumbar vertebrae height, and then collected the abdominal aorta (Ao). The collected Ao were vertically incised along the major axis of the blood vessel, beginning at the branching point of the renal artery, and separated into the ventral and dorsal sides. The removed ventral sides of the Ao were photographed from the intravascular lumen using a digital camera. Using the images, the horizontal and vertical diameters of the sites of confluence with the CT, SMA, and IMA were measured. In addition, the left/right deviation of the sites of confluence from the center line along the vertical axis of the vessels that passed through the midpoint between both sides of the branching point of the renal artery were measured. We also measured the branching points of the CT, SMA, IMA, and left renal artery (LtRA) using the common iliac artery (CoI) as the point of origin, and calculated the distances between the branching points (Fig. 1). These measurements were then analyzed using statistical software (GraphPad Prism version 7.0a for Mac, GraphPad Software, CA; www. graphpad.com). A $p$-value of less than 0.05 (two-tailed) was considered to indicate a statistically significant difference between two groups. An $r$-value of greater than 0.3 was considered to indicate a significant correlation.

\section{Results}

Comparison of the branching point of the IMA from the Ao and the vertebral heights indicated that the branching points of the IMA were distributed from the middle third of the L2 to the middle third of the L4, and that branching occurred at the L3 in $47 \%$ of the cadavers. Of these, the branching from the lower third of the L3 was the most frequent $(25 \%)$, but the majority arised from levels other than the L3 (Fig. 2).

Measurements of the branching points of the CT, SMA, and IMA indicated that the vertical diameters were $4.86 \pm 1.64 \mathrm{~mm}, 6.38 \pm 1.52 \mathrm{~mm}$, and $2.15 \pm 0.15 \mathrm{~mm}$, whereas the horizontal diameters were $7.38 \pm 1.86 \mathrm{~mm}$, $8.65 \pm 1.77 \mathrm{~mm}$, and $3.29 \pm 0.78 \mathrm{~mm}$ for the CT, SMA, and IMA, respectively (Table 1). Although the vertical diameters of the vessels demonstrated significant differences (all $p<0.0001$ ), only the CT and SMA showed a significant positive correlation (vertical: $r=0.55, p=$ 0.0013; horizontal: $r=0.44, p=0.0139$ ).
The vertical-horizontal ratios were $0.655 \pm 0.156$, $0.736 \pm 0.112$, and $0.636 \pm 0.117$, for the CT, SMA, and IMA, respectively, which indicate that all vertical diameters were significantly longer than the horizontal diameters. However, the vertical-horizontal diameter of the SMA was significantly different from those of the CT $(p=0.0416)$ and IMA $(p=0.0095)$ and was closer to an elliptical shape (Fig. 3). In addition, although the vertical-horizontal ratio of the SMA had a significant positive correlation to that of the CT $(r=0.507, p=0.003)$, the IMA did not correlate with either the CT $(r=-0.054, p=$ $0.772)$ or the SMA ( $r=0.077, p=0.681$; Fig. 4$)$.

Our analysis of the left/right deviation from the center line of the Ao to the confluence points of the CT, SMA, and IMA indicated that there was leftward deviation of the CT in $80 \%$ of the cadavers, of the SMA in $60 \%$ of the cadavers, and of the IMA in $71 \%$ of the cadavers. The average leftward deviation was $2.27 \pm 2.65 \mathrm{~mm}$ for the CT, $0.43 \pm 2.11 \mathrm{~mm}$ for the SMA and $1.04 \pm 2.3 \mathrm{~mm}$ for the IMA (Table 2).

Between the CT and CoI, the CT-SMA distance was $16.2 \pm 3.3 \mathrm{~mm}$, the SMA-Lt-RA distance was $15.6 \pm 8.2$ $\mathrm{mm}$, the Lt-RA-IMA distance was $54.3 \pm 8.9 \mathrm{~mm}$, and the IMA-CoI distance was $45.1 \pm 9.6 \mathrm{~mm}$. The ratios of each of the distances to the CT-CoI distance were $12.5 \%$ $\pm 2.6 \%, 10.8 \pm 6.0 \%, 41.9 \% \pm 7.1 \%$, and $34.1 \% \pm 4.7 \%$, for CT-SMA, SMA-LtRA, LtRA-IMA, and IMA-CoI, respectively. In cases in which the Lt-RA branching point was not measured, the SMA-IMA distance was $70.4 \pm$ $8.1 \mathrm{~mm}$, the ratio of the SMA-IMA to CT-CoI distance was $53.3 \% \pm 4.1 \%$, and the relative distance ratios for CT-SMA,SMA-IMA,IMA-CoI were 13\%, 53\%, 34\% , respectively (Fig. 5).

The CT-SMA distance and the SMA-IMA distance did not have a significant positive correlation to the actual measurements and the CT-CoI ratio (distance: $r=-0.0965, p=0.606$, ratio: $r=-0.0724, p=0.709$ ). However, the SMA-LtRA distance demonstrated a significant negative correlation with the actual LtRA-IMA distance measurements $(r=-0.513, p=0.032)$. The SMA-LtRA distance also exhibited weakly negative but statistically insignificant correlations with the CT-CoI distance ratio $(r=-0.250, p=0.0190)$. Compared to SMA-LtRA and LtRA-IMA, CT-SMA and SMA-IMA had less variation in their actual measurements and ratios to CT-CoI (Fig. 6).

\section{Discussion}

Previously, the IMA was thought to arise from the L3 ${ }^{1}$. Kakihara et al. ${ }^{2)}$ previously analyzed 3,182 abdominal angiographs, and reported that in all cases the IMA arised slightly to the left of the center of the Ao at the $\mathrm{L} 3$, and as a result the L3 is currently used as a landmark when performing angiographs of the IMA. However, 


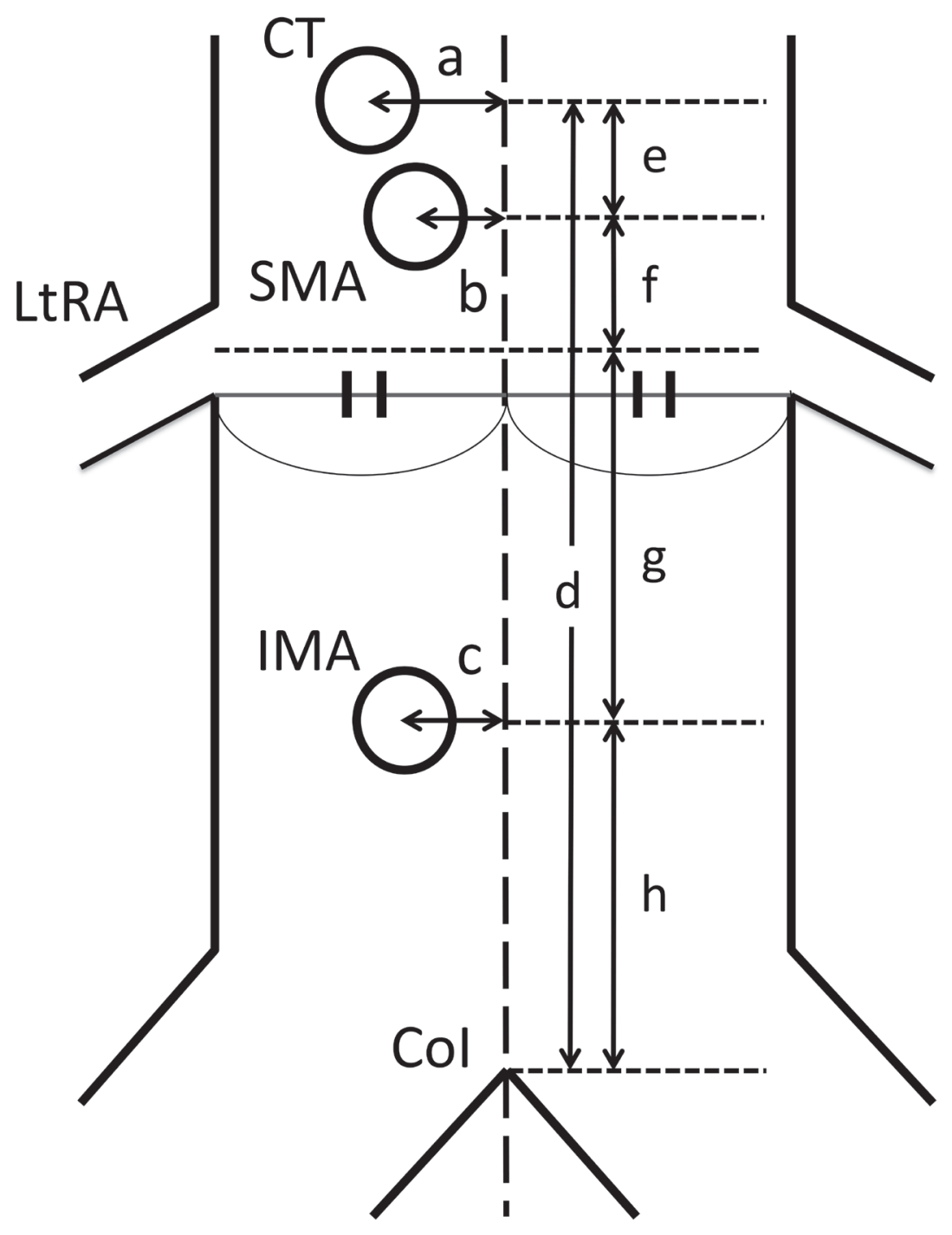

Fig. 1. A schematic diagram of measuretd items. The horizontal distance from the center line of the Ao to the confluence points of the CT (a), SMA (b), and IMA (c). The vertical distance between the confluence points of the CT and CoI (d), CT and SMA (e), SMA and LtRA (f), LtRA and IMA (g), and IMA and CoI (h).

Kahn et al. ${ }^{3)}$ reported that the branching point of the IMA was at the L3 only in approximately $66 \%$ of all cases, and that among these $27 \%$ occurred in the lower third of the L3. However, in approximately $30 \%$ of cases the IMA branches from locations other than the L3, and in our present study it arose at the L3 in approximately half the cases, with approximately $25 \%$ (the largest group) arising at the lower third of the L3, and grater than half of the cases branching at locations other than the L3. Kornreich et al. ${ }^{4}$ ) reported that arteries tend to elongate with age owing to arteriosclerosis, and that decreased vertebral height and intervertebral disk thickness as a result of osteoporosis and other conditions cause the bifurcation site of the common iliac artery to shift caudally. In our present study, many of the subjects were elderly individuals in their $80 \mathrm{~s}$. Therefore, caution is required when using lumbar vertebral height as a landmark during imaging of the IMA.

In their study of the relative locations of the branching sites of the branches of the abdominal aorta, Pennington et al.5) reported that although there were some weak correlations in the distances between the CT, SMA, and RA, the distances between their branching points and the branching point of the IMA were not correlated. In our present study, the CT-SMA distances and ratios to the CT-CoI distance showed the least variations, and although the SMA-IMA, SMA-LtRA, Lt-RA-IMA, and IMA-CoI distances showed a large amount of variation, there was little variation in the ratios of CT-CoI to SMA-IMA and to IMA-CoI. Conversely, ratios of 


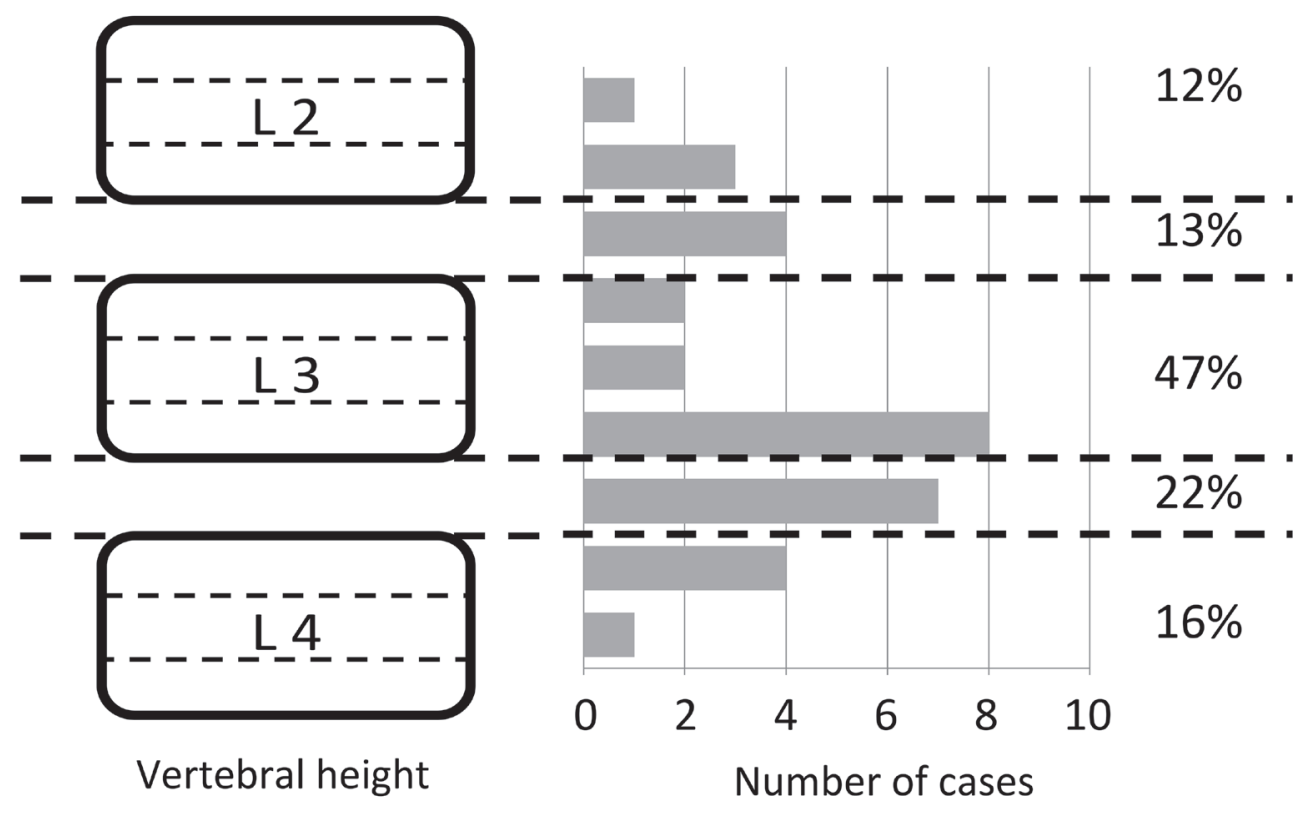

Fig. 2. Comparison of the branching points of the IMA from the Ao and the vertebral heights.

Table 1. Result of measurement of each branching points.

\begin{tabular}{lccc}
\hline & $\begin{array}{c}\text { Vertical diameter } \\
(\mathrm{mm})\end{array}$ & $\begin{array}{c}\text { Horizontal diameter } \\
(\mathrm{mm})\end{array}$ & $\begin{array}{c}\text { Vertical/Horizaontal } \\
\text { ratio }\end{array}$ \\
\hline CT & $4.86 \pm 1.64$ & $7.38 \pm 1.86$ & $0.655 \pm 0.156$ \\
SMA & $6.38 \pm 1.52$ & $8.65 \pm 1.77$ & $0.736 \pm 0.112$ \\
IMA & $2.15 \pm 0.15$ & $3.29 \pm 0.78$ & $0.636 \pm 0.117$ \\
\hline
\end{tabular}

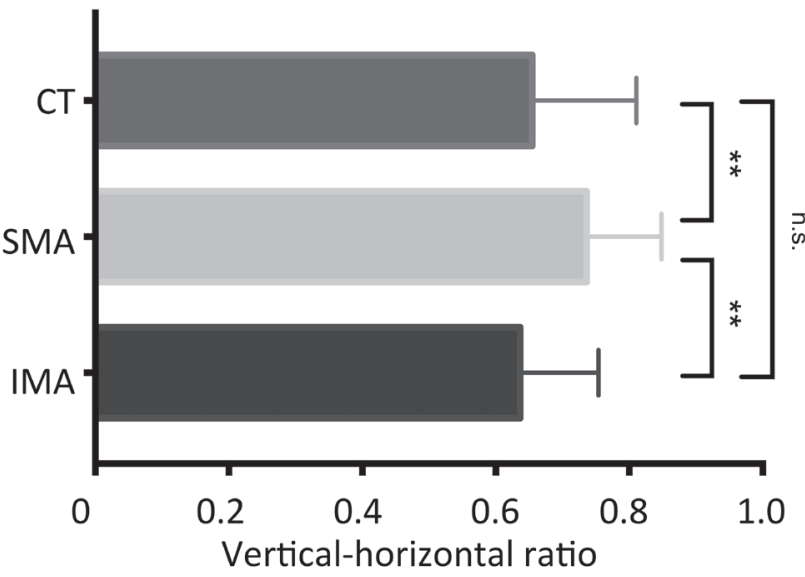

Fig. 3. The vertical-horizontal ratios each of the CT, SMA, and IMA.

CT-CoI to SMA-LtRA and to LtRA-IMA showed a large amount of variation. The ratios of CT-CoI to CT-SMA and to SMA-IMA as well as both actual measurements demonstrated no correlations, but the ratio of CT-CoI to the measured distances between the SMA-LtRA and

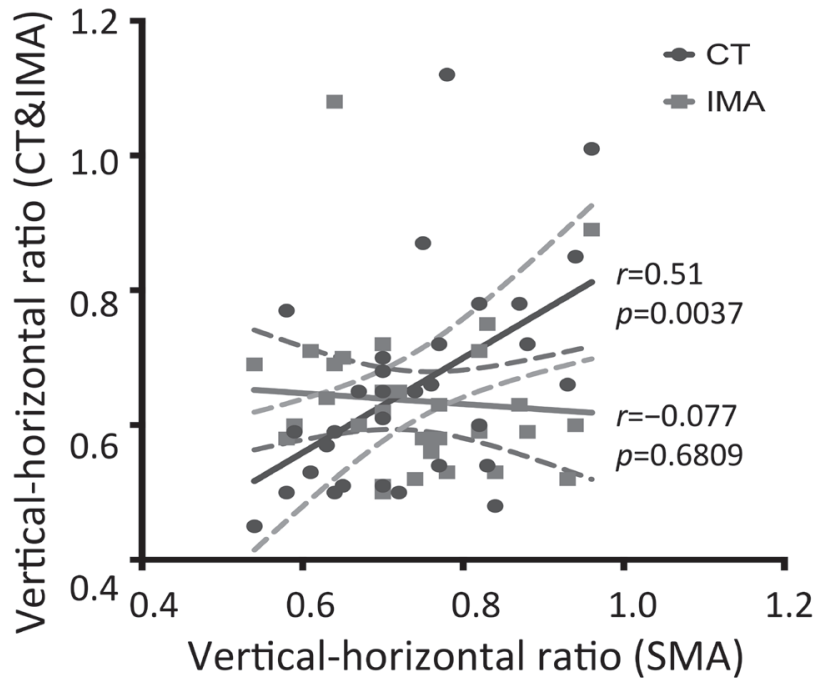

Fig. 4. Correlation of the vertical-horizontal ratio of the SMA to the CT and IMA. The vertical-horizontal ratio of the SMA had a significant positive correlation to that of the CT (black line), while the IMA did not correlate to the SMA (gray line).

LtRA-IMA showed relatively little variation. Pennington et al.5) reported that, similar to the findings in our present study, variations in the RA and IMA distance data were larger than those of the CT-SMA distance, SMA-RA distance, and the distance on both sides of the RA. In addition, as branching abnormalities, such as duplicate renal arteries exist in over $30 \%$ of cases ${ }^{6}$, it appears difficult to use the renal artery as a landmark when imaging 
Table 2. Horizaontal deviation from the Ao center line of the each branching points.

\begin{tabular}{lccccc}
\hline & Deviate to left (n) & $\begin{array}{c}\text { Distance from center } \\
\text { line }(\mathrm{mm})\end{array}$ & Deviate to right (n) & $\begin{array}{c}\text { Distance from center } \\
\text { line }(\mathrm{mm})\end{array}$ & On the center line (n) \\
\hline CT & 24 & $3.1 \pm 1.99$ & 5 & $1.91 \pm 1.26$ & 1 \\
SMA & 18 & $1.78 \pm 1.21$ & 12 & $1.6 \pm 1.47$ & 0 \\
IMA & 22 & $2.02 \pm 1.38$ & 7 & $2.16 \pm 1.76$ & 1 \\
\hline
\end{tabular}

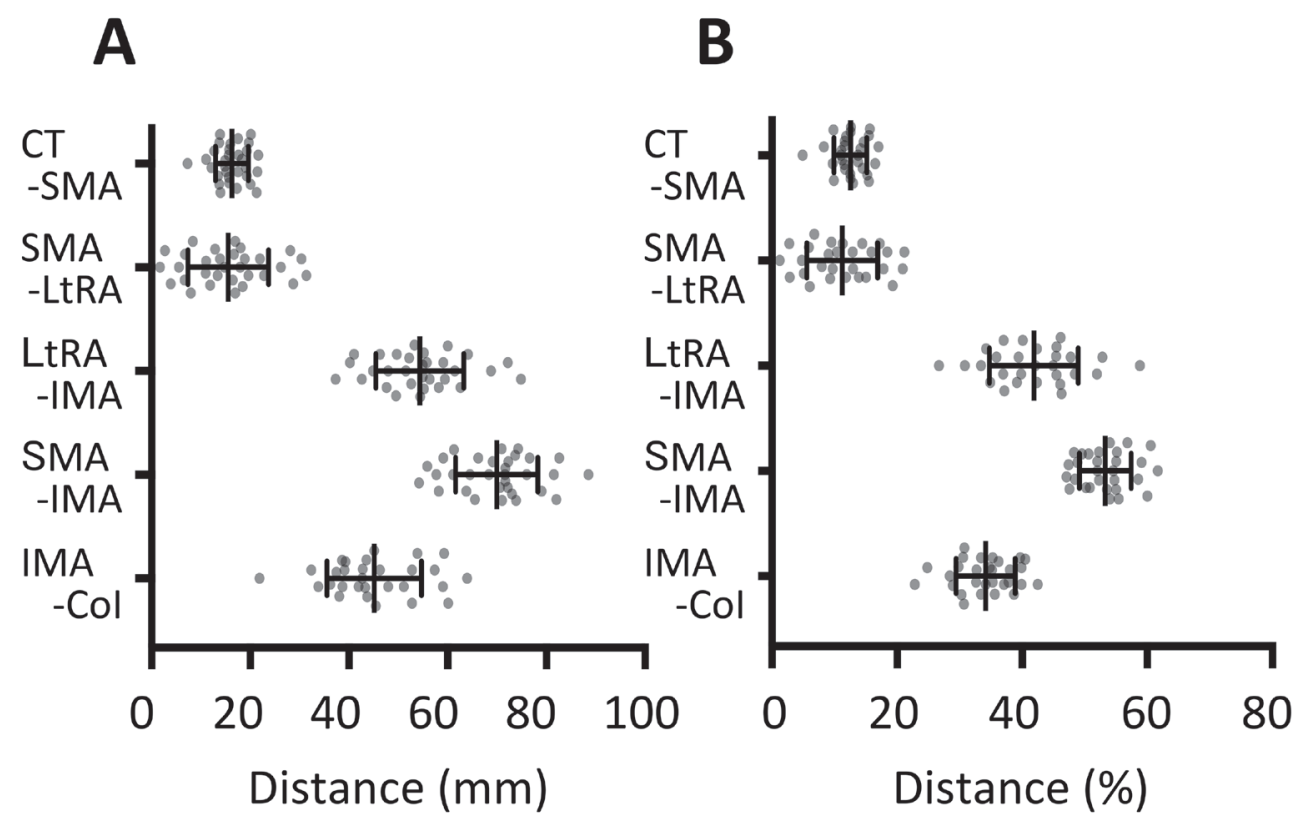

Fig. 5. The distances between each branching points showed a large amount of variation except of the CT-SMA distance (5A). There was a little variability in the ratio data for branching point distances relative to CT-CoI for CT-SMA, SMA-IMA, and IMA-CoI (5B).

the IMA.

Although there was variability in the actual measurement data, there was little variability in the ratio data for branching point distances relative to CT-CoI for CT-SMA, SMA-IMA, and IMA-CoI, with the ratios of $13 \%, 53 \%$ and $34 \%$, respectively.

Our investigation of the left/right deviations of the branching sites of the CT, SMA, and IMA from the central axis of the aorta showed that in the subjects in our study, all CT, SMA, and IMA branching points deviated from the central axis of the aorta slightly to the left, with the SMA closest to the center, followed by the IMA, and the CT farthest from the central axis. In their study of the relative locations of the branching points of the SMA and CT, Ozan et al. ${ }^{7}$ ) reported that in $73.3 \%$ of cases, the CT is located at the upper left of the SMA. This same relative positioning was reported in $60 \%$ of the cases studied by Michniewicz et al. ${ }^{8)}$, in $80 \%$ of the cases studied by Takahashi et al.9), and in $90 \%$ of the cases in the present study. In their study of the SMA, Michniewicz et al. $\left.{ }^{8}\right)$ reported that there was a deviation to the left in $42.2 \%$ of the cases, whereas we found a deviation to the left in $60 \%$ of our cases. In their study of the IMA, Maga et al. ${ }^{10)}$ reported that there was a deviation to the left in $88.7 \%$ of the cases, which was similar to the $90 \%$ found in our present study. Tandler11) reported that development of the aorta and rotation of the primitive gut in the fetal stage are associated with the left/right deviations and relative locations of the branching sites. As a result, the foregut, which branches off the $\mathrm{CT}$ at approximately 5 weeks after gestation, rotates 90 degrees clockwise relative to the axis of the body when observed from a cranial view. At this time, as the primitive spleen shifts left and to the rear, the point of confluence of the CT then shifts to the left. In contrast, the midgut, which is controlled by the SMA, rotates 270 degrees counterclockwise on an axis along the vitello-intestinal duct, which runs parallel to the SMA, and this then keeps the left/right deviation to a minimum. When the hindgut, which is controlled by the IMA, rotates counterclockwise to the midgut around the aforementioned axis along the vitello-intestinal duct, the entire hindgut lengthens and shifts to the left, which is thought to result in a shift of the site of confluence of the IMA also to the left. 

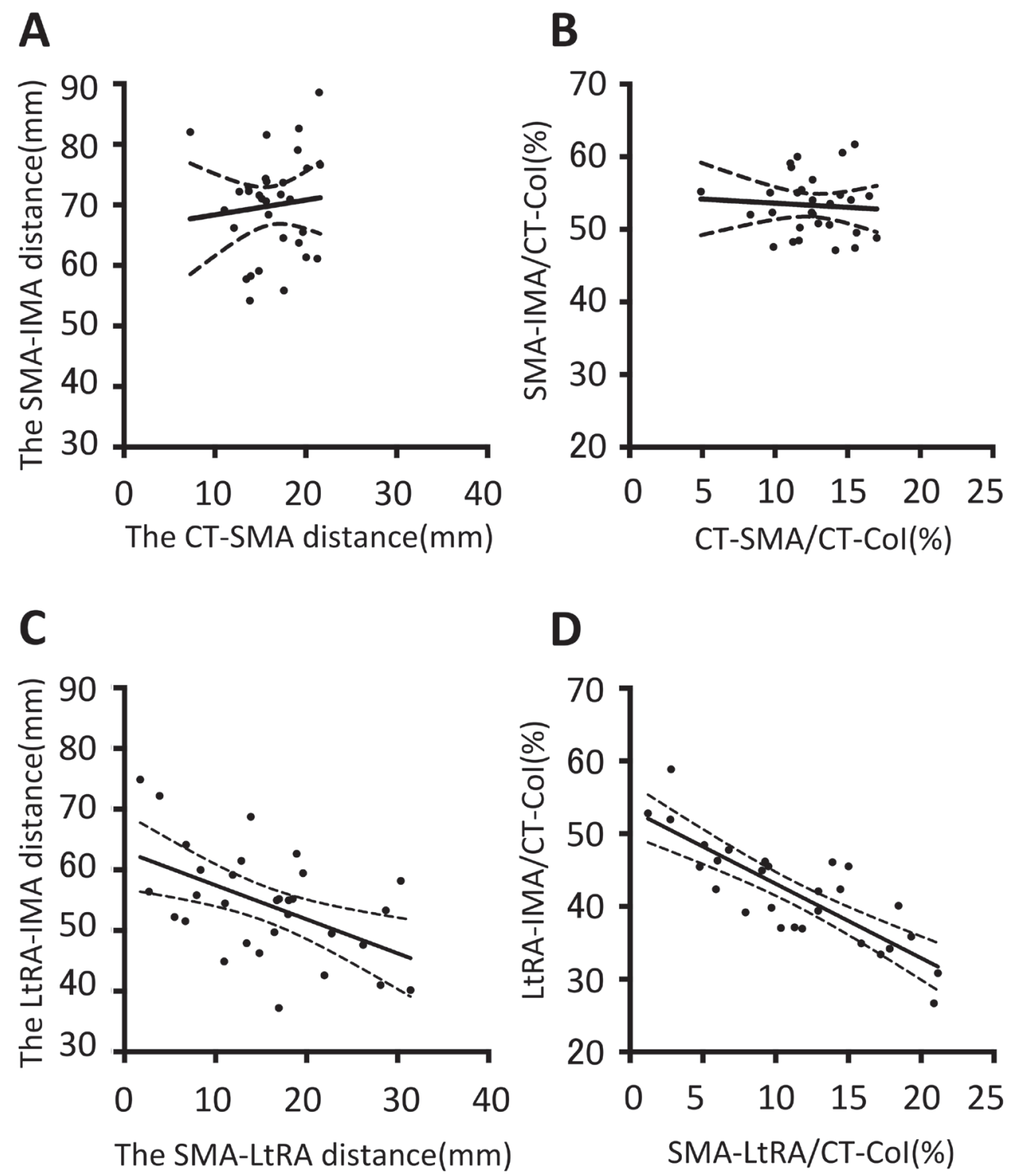

Fig. 6. Correlations between the distances between the aortic landmarks. Although CT-SMA and SMA-IMA did not have positive correlation to the actual measurement (6A) and the ratio to CT-CoI (6B), CT-SMA and SMA-IMA had less variation than that of SMA-LtRA and LtRAIMA in their actual measurements (6C) and ratios to CT-CoI (6D).

In their study of the actual measurements and morphology of the branching points of the CT, SMA, and IMA, Pennington et al. ${ }^{5)}$ reported that the horizontal diameters were $6.4 \pm 1.1 \mathrm{~mm}$ for the CT, $7.3 \pm 1.4 \mathrm{~mm}$ for the SMA, and $3.3 \pm 0.7 \mathrm{~mm}$ for the IMA, indicating that, as found in the present study, the IMA has a significantly smaller horizontal diameter than the CT and SMA. However, the authors did not measure the vertical diameters. In a study by Takahashi et al.9), the IMA was not measured, but the horizontal/vertical diameters were 8.1 $\pm 3.4 \mathrm{~mm} / 7.4 \pm 2.6 \mathrm{~mm}$ for the CT and $7.4 \pm 2.6 \mathrm{~mm} / 7.6$ $\pm 2.4 \mathrm{~mm}$ for the SMA. In our present study, we found that the horizontal diameter was larger than the vertical diameter in the CT and SMA. Although we found that the branching points of the CT and SMA correlated with the vertical diameter and horizontal diameter ratios in this study, the reason for this remains unknown. In their study of the branching point of the IMA, Kahn et al. ${ }^{3)}$ reported that there was a large variation in the data of the diameter of the branching points of the left colic artery blood supply zone. They found that if the left colic artery is affected only by the blood flow of the descending colon, then the diameter of the branching point is small, and that in cases in which the area that is affected by the blood flow extends to the transverse colon and splenic flexure, the diameter of the IMA branching point is large. These 
data suggest that the amount of blood flow in the IMA is associated with the diameter of the branching point. However, as the cases in our present study were cadavers, we were not able to investigate the association between blood supply zones and branching points.

In cases in which contrast-enhanced abdominal computed tomography is performed prior to an abdominal angiogram, 3D reconstruction takes time. Thus, when selecting the catheter size, the diameters of the branching points are measured in horizontal sections. However, as the horizontal diameter of the branching points is significantly larger than the vertical diameter when measured in horizontal sections, cannulation may be impossible owing to the fact that the catheter actually used has a vertical diameter that is smaller than the horizontal diameter. Therefore, the catheter diameter must be selected based on the vertical diameter rather than the horizontal diameter. As the diameter of the branching point of the IMA is significantly smaller than those of the branching points of the CT and SMA in some cases, it is advisable to use the vertical diameter when measuring the IMA. In cases in which abdominal angiography is performed first and in cases of extreme emergency, the use of a catheter with a diameter of no more than approximately $2 \mathrm{~mm}$ (approximately $6 \mathrm{Fr}=1.98 \mathrm{~mm}$ ) will enable imaging of the CT, SMA, and IMA with a single catheter.

As mentioned above, when imaging the IMA, great care must be given when using the L3 as a landmark in elderly patients. In such cases, the SMA should be imaged first because its location can be identified somewhat easily in relation to nearby organs that are located in a central position, and then the CT-SMA distance should be measured using contrast enhanced CT in the region to the upper left. Shifting the catheter in a caudal direction approximately four times this distance and then searching for the IMA branching point slightly to the left of the center will enable quick and safe contrast enhanced imaging. This will then reduce the amount of contrast medium used, lowering the patient's exposure to $\mathrm{X}$-rays and therefore minimizing the physical effects on the patient.

\section{Conclusions}

Cross sections of the sites of branching of the CT, SMA, and IMA often showed an elliptical morphology, but in some cases the SMA was closer to a round morphology. The IMA is significantly narrower and its vertical diameter is smaller than the other vessels. As a result, the vertical diameter of the IMA should be taken into consideration when selecting a catheter. When imaging the IMA, the branch is often at the lower third of the L3, but in the elderly, who may have age-related changes in their spines and abdominal aortas, caution is necessary. In cases in which it is difficult to image the IMA, a contrast-enhanced image of the CT and SMA should first be taken. The IMA branch is likely to be caudal and slightly to the left of the SMA branching point, which is a distance equivalent to four times that of the CT-SMA distance. Although the RA is relatively easy to image, it is difficult to use the RA as a landmark because its distance from all branching points varies greatly.

\section{Acknowledgement}

The authors wish to thank Mr. Koichi Koyama, Mr. Kenta Nagahori, Ms. Miyuki Kuramasu, Ms. Yuki Ogawa, Ms. Keiko Kuwana, and Ms. Yuka Kobayashi for their excellent technical and secretarial assistance. Finally, the authors give their heart-felt appreciation to the body donors and their families.

\section{References}

1) Fukuda $\mathrm{T}$ : Anatomy of aorta, Peripheral artery. Jpn J Intervent Radiol. 2010; 25:421-6 (in Japanese).

2) Kakihara N, Takahashi S, Tsuchihashi $Y$, et al: A rare case of abnormal inferior mesenteric artery. Jpn J Gastroenterol Surg. 2001; 34:1442-6.

3) Kahn P, Abrams HL: Inferior mesenteric arterial patterns. An angiographic study. Radiology. 1964; 82:429-42.

4) Kornreich L, Hadar H, Sulkes J, Gornish M, Ackerman J, Gadoth $\mathrm{N}$ : Effect of normal aging on the sites of aortic bifurcation and inferior vena cava confluence: a CT study. Surg Radiol Anat. 1998; 20:63-8.

5) Pennington N, Soames RW: The anterior visceral branches of the abdominal aorta and their relationship to the renal arteries. Surg Radiol Anat. 2005; 27:395-403.

6) Sampaio FJB, Passos MARF: Renal arteries: anatomic study for surgical and radiological practice. Surg Radiol Anat. 1992; 14:113-7.

7) Ozan H, Alemdaroglu A, Sinav A, Gümüsalan Y: Location of the ostia of the renal arteries in the aorta. Surg Radiol Anat . 1997; 19:245-7.

8) Michniewicz-Nowak M, Kosiński H: Skeletopy of the origin of major branches of the abdominal aorta and of bifurcation of the aorta. Foria Morphol(Warsz) . 1986; 45:262-8.

9) Takahashi $T$, Takeuchi $\mathrm{K}$, Ito $\mathrm{T}$, Ito $\mathrm{M}$ : Positinal relationships among the celiac trunk, superior mesenteric artery, and renal artery observed from the intravascular space. Surg Radiol Anat. 2013; 35:411-7.

10) Maga P, Kózka M, Pityński K, Chałupczak P, Skawina A: The anatomical variations of the inferior mesenteric artery and its branches in human fetus. Folia Morphol (Warsz). 1992; 51:313-8.

11) Tandler J: Entwicklungsgeschichte der menschlichen Darmarterien. Anat Hefte. 1903; 23:189-209. 